\title{
Synergistic Effect of Bazedoxifene and PARP Inhibitor in the Treatment of Ovarian Cancer Regardless of BRCA Mutation
}

\author{
RUIJIE ZHANG ${ }^{1,2}$, TIFFANY WANG ${ }^{2}$ and JIAYUH LIN ${ }^{2}$ \\ ${ }^{1}$ Department of Thoracic Surgery, Tongji Hospital, Tongji Medical College, \\ Huazhong University of Science and Technology, Wuhan, P.R. China; \\ ${ }^{2}$ Department of Biochemistry and Molecular Biology, \\ University of Maryland School of Medicine, Baltimore, MD, U.S.A.
}

\begin{abstract}
Background/Aim: Poly (ADP-ribose) polymerase inhibitors (PARPis) are one of the targeted therapies proven to treat breast cancer gene (BRCA)-mutant ovarian cancer. Because most ovarian cancers are BRCA wild-type, it is necessary to extend the usage of PARPis. In the present study, we combined the PARPi, talazoparib, and the IL-6 inhibitor, bazedoxifene, for the treatment of human ovarian cancer cells. Materials and Methods: The human ovarian cancer cell lines, SKOV3, UWB1.289 (BRCA1-null) and OV75, were treated with talazoparib and bazedoxifene, as monotherapy or combination treatment. The effects of treatment on cell viability, migration, growth and colony formation were examined. Western blot was used to investigate pathways that may be involved in the antitumor effects of the two agents. Results: The combination of talazoparib and bazedoxifene showed synergistic inhibition of cell viability, cell migration, cell growth, and cell colony formation on all the studied cell lines. The expression of $p-A K T, c-m y c, p-E R K, E R \alpha$ was inhibited, and $\gamma-H 2 A X$ expression was induced. Conclusion: Combined inhibition of PARP and IL-6 may be an efficacious treatment for ovarian cancer, independently of BRCA mutation status.
\end{abstract}

Ovarian cancer is the most lethal gynecological cancer and the fifth leading cause of cancer-related death in women in the USA (1). In 2019 alone, there were approximately 22,530 new

This article is freely accessible online.

Correspondence to: Jiayuh Lin, Department of Biochemistry and Molecular Biology, University of Maryland School of Medicine, Baltimore, MD, U.S.A. Tel: +1 4107067469, Fax: +1 4107068297, e-mail: JLin@som.umaryland.edu

Key Words: PARP, talazoparib, IL-6 inhibitor, bazedoxifene, BRCA, ovarian cancer. cases of ovarian cancer resulting in 13,980 deaths (2). The standard treatment for ovarian cancer is radical surgery with adjuvant chemotherapy and taxane combined with platinum as the first line approach (3). Surgical techniques and chemotherapy have been well-studied in the effort to improve long-term survival of ovarian cancer (4). However, the fiveyear survival rate has not increased significantly in the past two decades (3). Thus, there is urgent need to investigate new pharmacologic interventions with a tolerable side effect profile, and targeted therapy is a promising approach (5).

One of these targeted therapies, oral PARPis, have already been used as maintenance therapy in the treatment of ovarian cancer (6). The mechanism of PARPis was first reported as synthetic lethality in cancer cells with BRCA mutations (79). PARPis prevent the repair of DNA single-strand breaks (SSB), hence promoting the conversion of SSB to doublestrand breaks (10). BRCA1 and BRCA2 are essential players in DSB repair through homologous recombination (HR) (79). Therefore, cancer cells with BRCA loss-of-function mutations would only have low-fidelity repair pathways, resulting in an increase in genomic instability, ultimately leading to tumor-specific cell death (7-10). PARPis have been established as an anticancer therapy in patients with BRCA mutations, including talazoparib, which was approved by the Food and Drug Administration (FDA) in 2018 (11).

However, the majority of ovarian cancer patients do not have BRCA mutations (12). Thus, new studies have been focused on the possibility of extending the use of PARPis for patients without BRCA mutations. It has been reported that in addition to DNA repair pathways, PARP inhibitors also activate other pathways such as phosphoinositide 3-kinase (PI3K)/AKT, estrogen, cyclinD1, nuclear factor kappa B (NF-kB), extracellular signal-regulated kinase (ERK), and pro-inflammatory cytokines, such as tumor necrosis factor$\alpha(\mathrm{TNF} \alpha)$, interleukin (IL)-6, interferon- $\gamma(\mathrm{IFN} \gamma)$, E-selectin, and intercellular adhesion molecule (ICAM-1) (13). It has been previously demonstrated that the combination of 
PARPis with AKT inhibitors or CyclinD1 silencing led to greater inhibitory effects on cancer cells $(14,15)$.

IL-6 is a pro-inflammatory cytokine secreted by many cell types, including tumor cells (16), stimulating the generation and growth of tumors. IL- 6 is secreted by most ovarian cancer cells and promotes tumor cell growth, migration, and invasion $(4,17,18)$. High levels of IL-6 can result in chemoresistance and are a marker for poor prognosis in ovarian cancer patients (19-21). Therefore, inhibiting IL-6 is a promising targeted therapy for ovarian cancer cells.

The IL-6/JAK/STAT3 is the main pathway activated by IL-6, which binds to the IL-6 receptor and GP130 to form a signaling complex, leading to tyrosine phosphorylation of STAT3 (p-STAT3) and activation of downstream target genes like c-Myc $(22,23)$. However, many other pathways can also be activated by IL-6, such as PI3K/AKT, MAPK/ERK, Wnt, and COX-2 (24). Moreover, IL-6 can also increase estrogen secretion, which in turn can induce higher IL-6 levels. Bazedoxifene, initially established as a third-generation selective estrogen receptor modulator, was found in our previous studies to inhibit IL-6/GP130 (25-29). We have previously shown that bazedoxifene has antitumor activity in breast cancer (23) and colon cancer (30) cells, while having a favorable side effect profile.

Together, PARPis and IL-6 inhibitors have the potential for therapeutically significant antitumor activity through interplay across several pathways, suggesting synergistic effects on ovarian cancer cells, therefore allowing usage of PARPis for a wider patient population. Hence, in the present study, the PARPi, talazoparib, and the IL-6 inhibitor, bazedoxifene, were investigated as a combination therapy for ovarian cancer, both BRCA-null and wild-type ovarian cancer cells, in vitro.

\section{Materials and Methods}

Chemicals. Talazoparib was purchased from MedKoo Biosciences (\#204710) and azedoxifene acetate was purchased from SigmaAldrich (Merck KGaA, Darmstadt, Germany; \#198481-33-3). Both were stored in DMSO (dimethyl sulfoxide; Sigma-Aldrich, Merck $\mathrm{KGaA})$ as $20 \mathrm{mM}$ at $-20^{\circ} \mathrm{C}$. 3-(4, 5-dimethylthiazol -2-yl)-2, 5diphenyltetrazolium bromide (MTT) was purchased from SigmaAldrich (\#298-93-1), and the stock concentration was stocked in $\mathrm{ddH}_{2} \mathrm{O}$ as $5 \mathrm{mM}$ at $-20^{\circ} \mathrm{C}$. Primary and secondary antibodies were purchased from Cell Signaling Technology.

Cell lines. Human ovarian cancer cell lines SKOV3 and UWB1.289 were purchased from American Type Culture Collection (Manassas, VA, USA). The OV75 cell line was kindly provided by Dr. Jocelyn Reader (University of Maryland School of Medicine Division of Gynecologic Oncology, Baltimore, MD, USA). The OV75 cell line was cultured in HOSE Media (31). The UWB 1.289 cells, a BRCA1null cell line (32), were cultured in a 1:1 mixture of RPMI-1640 medium (Corning Inc., Corning, NY, USA; cat. no. 10-040-CV) with mammary epithelial cell growth medium (MEGM, Lonza Pharma \&
Biotech Inc., Basel, Switzerland; cat. no.CC-3150) with 3\% fetal bovine serum (FBS, Sigma-Aldrich; Merck KGaA; cat. no.F2442). The SKOV3 cell line was cultured in Dulbecco's Modified Eagle Medium (DMEM, Corning, Inc.; cat. no. 10-013-CV) supplemented with $10 \%$ FBS and $1 \%$ penicillin/streptomycin (ThermoFisher Scientific Inc., Waltham, MA, USA; cat. no.15140122). The medium was replaced twice a week. All cell lines were maintained in a humidified atmosphere with $5 \% \mathrm{CO}_{2}$ at $37^{\circ} \mathrm{C}$.

Measurement of interleukin-6. Cells were seeded in a 6-well plate at a density of $70 \%$ per well. The cell-free culture supernatant was collected the next day. IL-6 levels were detected in the supernatant using enzyme linked immunosorbent assay (ELISA) at OD=450 nm (Central Laboratory of the Netherlands Red Cross Blood Transfusion Service, Amsterdam, Netherlands). Each assay detects cytokine concentrations as low as $5 \pm 7 \mathrm{pg} / \mathrm{ml}$.

Western blot analysis. The ovarian cancer cells were seeded in 10-cm plates at a cell density of $70 \%$ and treated with talazoparib $(0.5 \mu \mathrm{M})$, bazedoxifene $(2.5 \mu \mathrm{M})$, combination treatment, or DMSO at $37^{\circ} \mathrm{C}$ overnight. Protein was extracted using ice cold lysis buffer (Cell Lysis Buffer, Cell Signaling Technology, Inc. cat. no. 9803S; 0.5\% 0.2 M PMSF, 0.5\% 0.2 M NaF, 0.5\% 0.2 M NaPP, 0.5\% 0.1 $\mathrm{M} \mathrm{Na}_{3} \mathrm{VO}_{4}$ and $4 \%$ 25X CP1). Protein concentration was determined using the Microplate BCA Protein Assay kit (Thermo Fisher Scientific, Inc.; cat. no. 23252). Proteins were separated by $10 \%$ SDS-PAGE gels and transferred to PVDF membrane under $350 \mathrm{~mA}$ for $110 \mathrm{~min}$. After blocking in $5 \%$ milk for $1 \mathrm{~h}$ at room temperature, membranes were incubated in primary antibodies overnight at $4^{\circ} \mathrm{C}$. After washing with Tris-buffered saline with $0.1 \%$ Tween-20 3 times for $15 \mathrm{~min}$, membranes were incubated with secondary antibody for $1.5 \mathrm{~h}$. Blots were imaged with the Storm scanner (Amersham Pharmacia Biotech Inc, Piscataway, NJ, USA) and Amersham Imager 680 (GE healthcare Inc, Mariborough, MA, USA).

All primary antibodies were purchased from Cell Signaling Technology, stored at $-20^{\circ} \mathrm{C}$, and diluted $1: 1000$ in $5 \%$ milk: pSTAT3 (Y705) (rabbit mAb, \#9131S), STAT3 (rabbit mAb, cat. \#4904S), p-AKT (rabbit mAb, \#4060S), AKT (rabbit mAb; \#4691S), phospho-histone H2A.X (rabbit mAb, \#2577S), phosphop44/42 MAPK (Erk1/2) (rabbit mAb, \#4370S), p44/42 MAPK (Erk1/2) (rabbit mAb, \#4569S), c-Myc (rabbit mAb, \#13987S), Estrogen Receptor $\alpha$ (D8H8) (rabbit mAb, \#8644S), GAPDH (rabbit $\mathrm{mAb}, \# 2118 \mathrm{~S})$. The secondary antibody was diluted 1:10000 in 5\% milk (Cell Signaling Technology, Anti-rabbit IgG HRP-linked antibody, \#7074).

MTT cell viability assay. Ovarian cancer cells were seeded in 96well microtiter plates with 3,000 cells and $100 \mu \mathrm{l}$ medium per well. Cells were treated with different drugs: talazoparib $(0.1 \mu \mathrm{M}$ for UWB1.289; $0.5 \mu \mathrm{M}$ for SKOV3; $0.5 \mu \mathrm{M}$ for OV75), bazedoxifene (5 $\mu \mathrm{M}$ for UWB1.289; $2.5 \mu \mathrm{M}$ for SKOV3; $2.5 \mu \mathrm{M}$ for OV75), combination treatment and DMSO after overnight incubation. After a 72 -h incubation at $37^{\circ} \mathrm{C}, 20 \mu \mathrm{MTT}$ was added to each well for an additional 4-h incubation. Then, $150 \mu \mathrm{l}$ of DMF solubilization solution was added into each well and incubated overnight in a shaker, protected from light, at room temperature. The absorbance was read at $\mathrm{OD}=595 \mathrm{~nm}$ using an EL808 Ultra Microplate Reader (BioTek, Winooski, VT, USA). The DMSO cells were set at $100 \%$ and the cell viability of drug-treated cells was determined relative to DMSO cells. Then, the combination index (CI) was determined 
by CompuSyn software (www.combosyn.com). The CI values indicate additive effect when equal to 1 , an antagonistic effect when $>1$, and a synergistic effect when $<1$, based on the theorem of ChouTalalay (33).

siRNA transfection. The SKOV3 and UWB1.289 cells were seeded in 96-well plates with 4,000 cells per well and transfected with GP130 siRNA (Cell Signaling Technology, \#6582s) or negative control siRNA (Cell Signaling Technology, \#6568s) at a final concentration of $50 \mathrm{nM}$ using Lipofectamine 2000 (Invitrogen, Carlsbad, CA, \#11668019) according to the manufacturer's instructions. Cells were transfected for $48 \mathrm{~h}$ and subsequently processed for the MTT assay, after which, cells were treated with DMSO or talazoparib $(1.0 \mu \mathrm{M}$ for UWB1.289; $0.5 \mu \mathrm{M}$ for SKOV3) for $72 \mathrm{~h}$.

Wound healing/cell migration assay. SKOV3 cells were seeded in a 6-well plate and cultured overnight until adherent and 100\% confluent. A $100-\mu l$ pipette tip was used to make a straight scratch in the monolayer of each well. Then, cells were washed with PBS twice and treated with talazoparib $(0.5 \mu \mathrm{M})$, bazedoxifene $(2.5 \mu \mathrm{M})$, combination treatment, or DMSO in culture medium. Images were captured at $0 \mathrm{~h}$ as well as after incubation at $37^{\circ} \mathrm{C}$ for $17 \mathrm{~h}$, when the wound of DMSO-control well healed. All images were captured by a Leica microscope with Leica Application Suite X (LAS X) software platform. Inhibition of migration was measured using ImageJ software (http://rsb.info.nih.gov/ij/) and calculated according to the formula: $100-$ [(final area/initial area) $\times 100 \%](34)$.

Cell growth assay. The same number of cells were seeded in 24well plates (SKOV3, $2 \times 10^{3}$ cells per well; UW1.289, $5 \times 10^{3}$ cells per well), cultured overnight at $37^{\circ} \mathrm{C}$, and treated with talazoparib $(0.005 \mu \mathrm{M}$ for UWB1.289; $0.5 \mu \mathrm{M}$ for SKOV3), bazedoxifene (2.5 $\mu \mathrm{M}$ for UWB1.289; $2.5 \mu \mathrm{M}$ for SKOV3), combination treatment, or DMSO. Cell number was counted on days 2, 4, 6 and 8 after treatment. At last, we plotted the growth curves and analyzed them.

Colony-forming assay. The same number of cells were seeded in 6well plates (SKOV3: $2 * 104$ cells per well, UW1.289: $5 * 103$ cells per well), cultured overnight at $37^{\circ} \mathrm{C}$, and treated with talazoparib (0.001 $\mu \mathrm{M}$ for UWB1.289; $1 \mu \mathrm{M}$ for SKOV3), bazedoxifene (5 $\mu \mathrm{M}$ for UWB1.289; $5 \mu \mathrm{M}$ for SKOV3), combination treatment, or DMSO. After a 48-h treatment, cells were incubated in fresh medium for 2-3 weeks (UWB1.289 for 3 weeks; SKOV3 for 2 weeks). Then, cells were washed with PBS, fixed with methanol for $30 \mathrm{~min}$, and stained with toluidine blue for $1.5 \mathrm{~h}$. The colonies were counted under a microscope (Olympus IX81, Tokyo, Japan).

Statistical analysis. Data were analyzed using GraphPad Prism 7 software (GraphPad Software Inc., San Diego, CA, USA) and all quantitative are expressed as mean \pm standard deviation (SD) of three independent experiments. Statistical significance was assessed using one-way and two-way ANOVA test with Tukey's test. Significance was set at $p<0.05$. One $(*)$, two $(* *)$, and three $(* * *)$ asterisks are used to indicate $p<0.05, p<0.01$ and $p<0.001$, respectively.

\section{Results}

IL-6 levels in human ovarian cancer cells. IL-6 levels were measured in ovarian cancer cells SKOV3, UWB1.289, and OV75 using ELISA. As shown in Figure 1, ovarian cancer

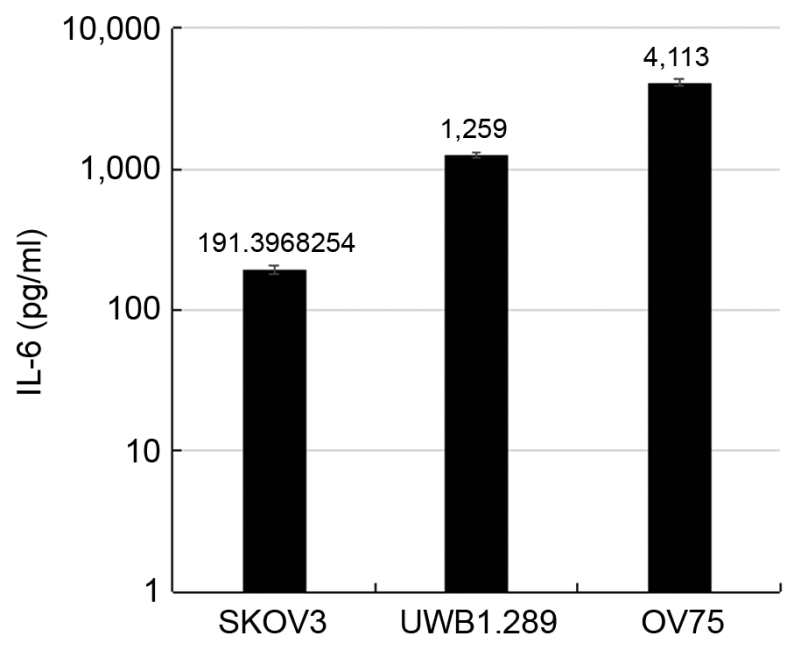

Figure 1. Interleukin (IL)-6 levels in human ovarian cancer cells.

cells expressed high levels of IL-6 (SKOV3, $191 \mathrm{pg} / \mathrm{ml}$; UWB1.289, 1259 pg/ml; OV75, 4113 pg/ml).

Bazedoxifene combined with talazoparib synergistically inhibited cell viability of human ovarian cancer cells. The effects of bazedoxifene and talazoparib on ovarian cancer cell viability were confirmed and compared to the combination treatment. As shown by MTT assay (Figure 2A), ovarian cancer cell viability was inhibited by talazoparib, bazedoxifene alone, as well as by the combination treatment in UWB1.289 [bazedoxifene (B), $87.3 \%$, B vs. DMSO (D), $p=0.0033$; talazoparib (T), 77.0\%, T $v s . \mathrm{D}, p<0.0001$; $\mathrm{B}+\mathrm{T}, 60.5 \%, \mathrm{~B} v s . \mathrm{B}+\mathrm{T}, p<0.0001$, T vs. $\mathrm{B}+\mathrm{T}$, $p=0.0006$ ], SKOV3 (bazedoxifene, 54.4\%, B vs. D, $p<0.0001$; talazoparib, 78.6\%, T vs. D, $p<0.0001$; $\mathrm{B}+\mathrm{T}$ : $42.9 \%$, B vs. $\mathrm{B}+\mathrm{T}$, $p=0.0003$, T vs. $\mathrm{B}+\mathrm{T}, p<0.0001$ ) and OV75 cells (bazedoxifene, $53.5 \%$, B vs. D, $p<0.0001$; talazoparib, $75.4 \%$, T $v s . \mathrm{D}$, $p=0.0009$; $\mathrm{B}+\mathrm{T}: 36.2 \%, \mathrm{~B}$ vs. $\mathrm{B}+\mathrm{T}, p=0.0081, \mathrm{~T}$ vs. $\mathrm{B}+\mathrm{T}$, $p<0.0001)$. Moreover, combination treatment induced greater inhibition of cell viability compared to monotherapy. Furthermore, the combination index (CI) of the two drugs was found to be $<1$ (UWB1.289, CI=0.4381; SKOV3, CI=0.80235; $\mathrm{OV75}, \mathrm{CI}=0.35628)$, demonstrating a synergistic effect of the two drugs.

According to our previous work, bazedoxifene is an inhibitor of IL-6, which requires IL-6 receptor and GP130 binding to form a signaling complex that activates target genes $(22,23,35)$. Thus, GP130 knockdown can inhibit cell viability. GP130 was knocked down by siRNA transfection, followed by MTT assay. The results showed that the cell viability of ovarian cancer cells was inhibited by GP130 knockdown (Figure 2B). When talazoparib was added, the combination treatment demonstrated a greater inhibitory effect than monotherapy in UWB1.289 [GP130-siRNA, 

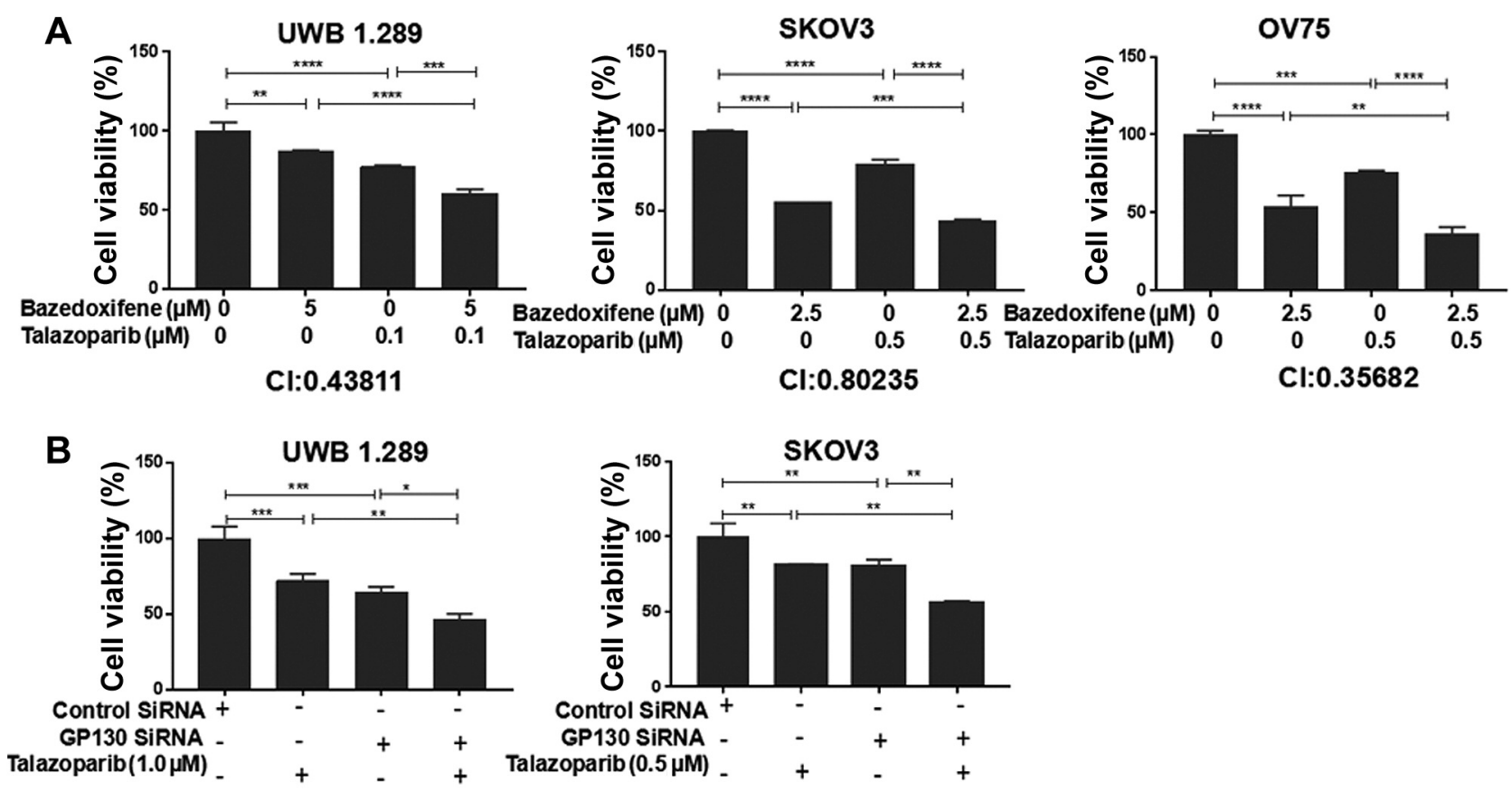

Figure 2. Effects of talazoparib, bazedoxifene, and their combination on cell viability. MTT assays were performed to evaluate cell viability. Cell viability of ovarian cancer cells was synergistically inhibited by the bazedoxifene and talazoparib combination (A). Transfection with GP130 siRNA followed by MTT assays (B). *p<0.05, **p<0.01, ***p<0.001 and $* * * * p<0.0001$.

64.4\%, GP130-siRNA(G) vs. Control-siRNA(C), $p=0.0002$; talazoparib, $71.9 \%$, T vs. C, $p=0.0009 ; \mathrm{T}+\mathrm{G}: 46.4 \%$, T vs. $\mathrm{T}+\mathrm{G}, p=0.0016, \mathrm{G} v s . \mathrm{T}+\mathrm{G}, p=0.0132]$ and SKOV3 cells (GP130- siRNA, $80.5 \%, G$ vs. C, $p=0.0054$; talazoparib, $80.7 \%$, T vs. C, $p=0.0057 ; \mathrm{T}+\mathrm{G}: 55.9 \%$, T $v s . \mathrm{T}+\mathrm{G}$ $p=0.0010, \mathrm{G} v s . \mathrm{T}+\mathrm{G} p=0.0012$ ).

Bazedoxifene combined with talazoparib inhibited cell migration of human ovarian cancer cells. The effects of bazedoxifene and talazoparib on cell migration were investigated using the wound healing assay on the SKOV3 cell line, but not UWB1.289, due to its monolayer phenotype unsuitable for this assay. Our results (Figure 3) showed that both bazedoxifene and talazoparib monotherapy inhibited cell migration of SKOV3 and the combination of these two drugs induced a greater inhibitory effect than monotherapy (SKOV3: bazedoxifene, $65.2 \%$, B vs. DMSO, $p<0.0001$; talazoparib, $61.5 \%$, T $v s$. DMSO, $p<0.0001$; B+T: $22.4 \%, \mathrm{~B}$ $v s . \mathrm{B}+\mathrm{T}, p<0.0001, \mathrm{~T} v s . \mathrm{B}+\mathrm{T}, p<0.0001)$.

Bazedoxifene combined with talazoparib inhibited cell growth of human ovarian cancer cells. Given that the combination of bazedoxifene and talazoparib synergistically inhibited the cell viability of ovarian cancer cells, we further analyzed the effect of the two drugs on cell growth. Our results (Figure 4) showed that either bazedoxifene or talazoparib inhibited cell growth of ovarian cancer cells and the combination of the two drugs demonstrated a greater inhibitory effect than monotherapy in UWB1.289 (DMSO, $265.7 \times 10^{3}$; bazedoxifene, $81.3 \times 10^{3}$, B vs. $\mathrm{D}, p<0.0001$; talazoparib, $50.3 \times 10^{3}$, T vs. D, $p<0.0001$; B+T: $9.0 \times 103$, B $v s . \mathrm{B}+\mathrm{T}, p<0.0001$, $\mathrm{T} v s . \mathrm{B}+\mathrm{T}, p<0.0001)$ and $\mathrm{SKOV} 3$ cells (DMSO, $114.3 \times 10^{3}$; bazedoxifene, $63.0 \times 10^{3}$, B vs. D, $p<0.0001$; talazoparib, $82.0 \times 10^{3}$, T vs. $\mathrm{D}, p<0.0001$; $\mathrm{B}+\mathrm{T}$ : $29.7 \times 10^{3}$, B vs. B+T, $p<0.0001$, T $\left.v s . \mathrm{B}+\mathrm{T}, p<0.0001\right)$.

Bazedoxifene combined with talazoparib inhibited cell colony formation of human ovarian cancer cells. Cell colony formation assay was performed to analyze the effect of talazoparib and bazedoxifene on colony formation in ovarian cancer cells, an important stem cell behavior which plays a significant role in tumor recurrence and metastasis $(36,37)$. The results (Figure 5) showed that talazoparib and bazedoxifene each individually inhibit colony formation of the ovarian cancer cells. The combination of the two drugs demonstrated a greater inhibitory effect than monotherapy of either drug (Figure 5) (UWB1.289: bazedoxifene, 49.4\%, B vs. $\mathrm{D}, p<0.0001$; talazoparib, 30.0\%, T vs. $\mathrm{D}, p<0.0001$; $\mathrm{B}+\mathrm{T}: 7.2 \%, \mathrm{~B}$ vs. $\mathrm{B}+\mathrm{T}, p<0.0001$, $\mathrm{T}$ vs. $\mathrm{B}+\mathrm{T}, p<0.0001$; SKOV3: bazedoxifene, $45.9 \%$, B vs. $\mathrm{D}, p<0.0001$; talazoparib, 32.1\%, T vs. D, $p<0.0001$; $\mathrm{B}+\mathrm{T}$ : $25.5 \%$, B $v s$. $\mathrm{B}+\mathrm{T}, p=0.0018, \mathrm{~T} v s . \mathrm{B}+\mathrm{T}, p=0.0186)$. Thus, talazoparib and 


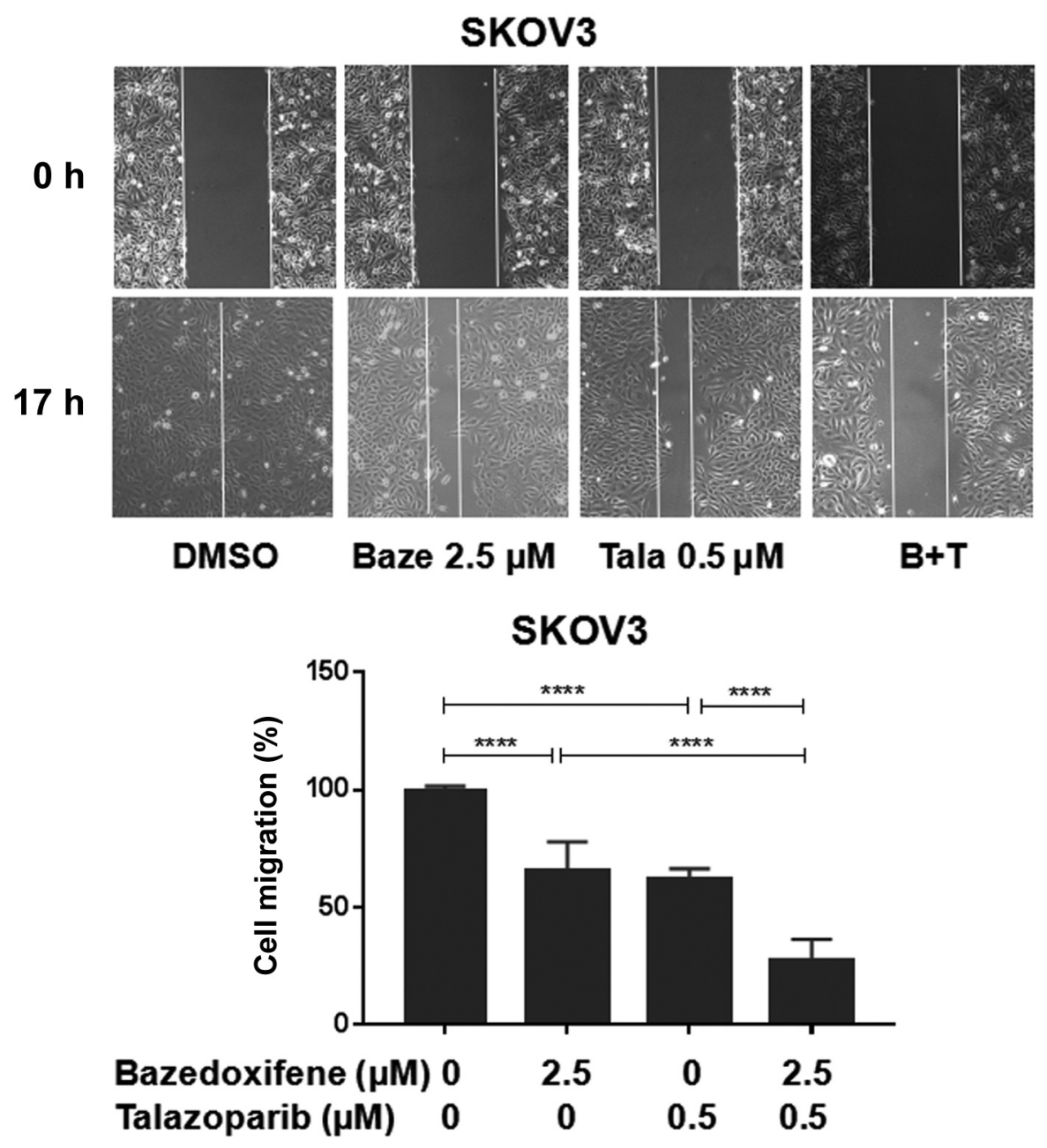

Figure 3. Effects of talazoparib and bazedoxifene monotherapy and combination treatment on cell migration. Wound-healing assays were performed to detect the migration ability of SKOV3 cells after talazoparib, bazedoxifene, combination, or DMSO treatment. $* * * p<0.001$ and $* * * * p<0.0001$.

bazedoxifene combination treatment has the potential to prevent ovarian cancer recurrence and metastasis.

Bazedoxifene combined with talazoparib synergistically inhibited the expression of targeted genes in human ovarian cancer cells. In order to investigate the possible pathways of interaction between talazoparib and bazedoxifene, western blot analysis was performed by us. According to our results (Figure 6), the expression of p-AKT, c-Myc, p-ERK and ER $\alpha$ was inhibited by the combination of the two drugs, while $\gamma \mathrm{H} 2 \mathrm{AX}$ expression, which is a biomarker for DNA double-strand breaks (38), was induced. The $p$-values of DMSO groups compared with $\mathrm{B}+\mathrm{T}$ groups were less than 0.05 (SKOV3: p-AKT, $p=0.0436$; c-Myc, $p=0.0087$; p-ERK, $p=0.0003 ; \mathrm{ER} \alpha, p=0.0332 ; \gamma \mathrm{H} 2 \mathrm{AX}$, $p<0.0001$. UWB1.289: p-AKT, $p=0.0179$; c-Myc, $p<0.0001$; ERK $p=0.001 ; \mathrm{ER} \alpha, p<0.0001 ; \gamma \mathrm{H} 2 \mathrm{AX}, p=0.0158)$.

\section{Discussion}

Ovarian cancer is one of the most common tumors in women in USA and has a high recurrence and mortality rate (3). More than half of patients are diagnosed with advanced ovarian cancer and there are approximately $75 \%$ of ovarian cancer patients will relapse due to intrinsic and acquired chemotherapy resistance, which lead to cancer recurrence. Thus, it is necessary to develop new treatment methods and drugs. Targeted therapies have been studied in ovarian cancer, demonstrating improved therapeutic efficacy and minimized side effects (39). Therefore, ovarian cancer research has been focused on discovering new targeted therapies (40).

PARPis are new class of antitumor therapy agents; they lead to the formation of double-strand breaks at replication forks, due to an accumulation of DNA single-strand breaks 

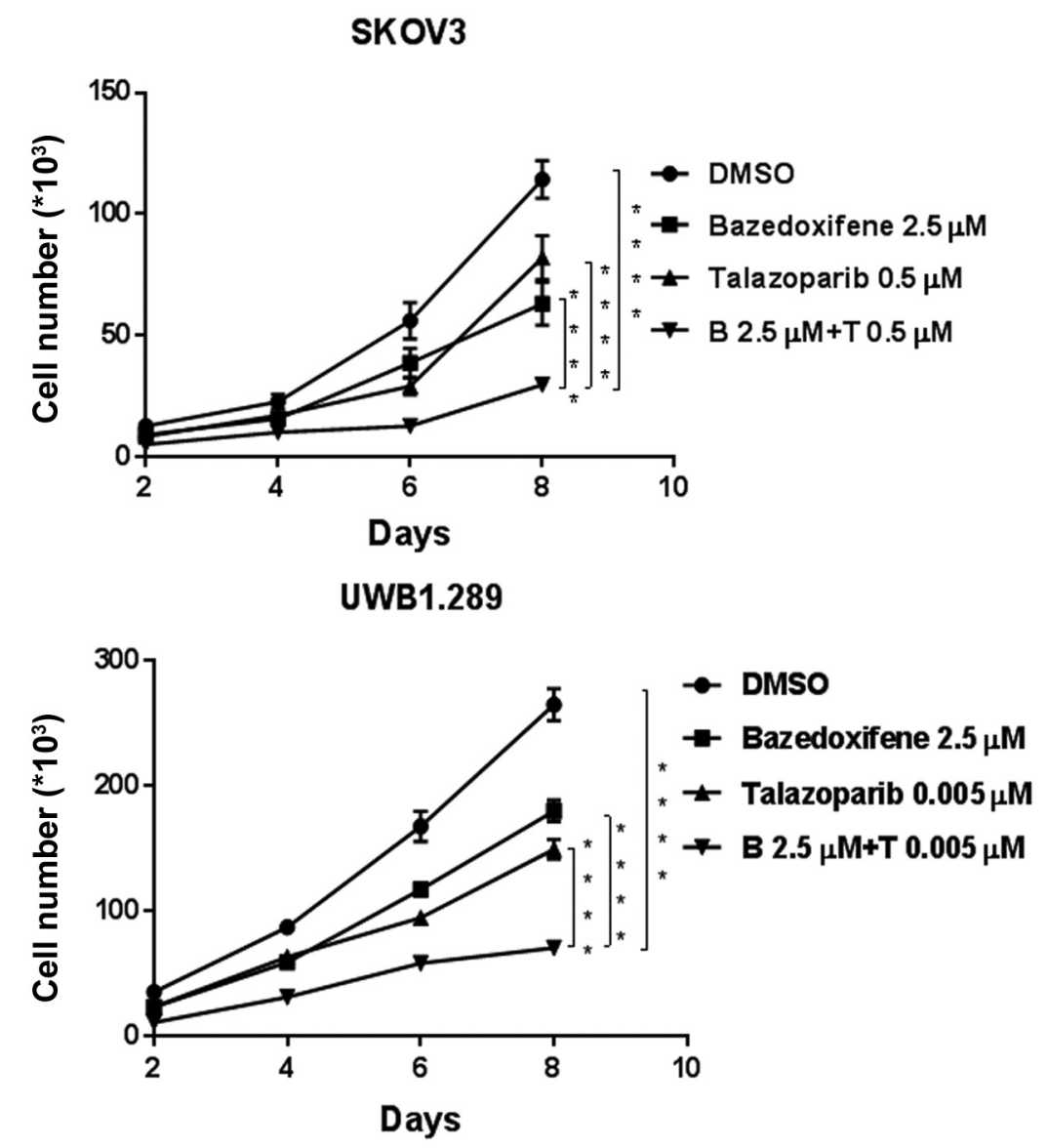

Figure 4. Effects of talazoparib, bazedoxifene and drug combination on cancer cell growth, $*^{*} p<0.01$ and $* * * * p<0.0001$. The cell growth of ovarian cancer cells was synergistically inhibited by combination treatment with bazedoxifene and talazoparib.

(41). PARPis have already been used as maintenance therapy in the treatment of BRCA-mutant ovarian cancer with good efficacy and a favorable side effect profile as well $(5,6,42,43)$. However, the majority of patients present with BRCA-proficient disease and are not eligible for this important class of drug (44). Therefore, researchers have been focusing on the use of PARPis in ovarian cancer with wild-type BRCA status.

During the last decade, the clinical use of PARPis has been further expanded to ovarian cancer patients without BRCA mutations. Olaparib (45) and niraparib (46) were approved by FDA as the maintenance therapy for complete or partial platinum-sensitive ovarian cancer, irrespective of the the BRCA status. Lynparza (47) and rucaparib (48) were also approved as maintenance treatment regardless of BRCA status. Additionally, it has been found that the combination of DNA methyltransferase 1 inhibitor (DNMTi) and talazoparib inhibited breast and ovarian cancer cells harboring either wild-type or mutant BRCA (44). Cyclin D1 silencing or AKT inhibitors have been shown to sensitize BRCA1 wildtype ovarian cancer cells to olaparib $(14,15)$, and the combination of carboplatin and olaparib demonstrated synergistic effect beyond BRCA-dependent synthetic lethality in high-grade serous ovarian cancer cell lines (49). Taken together, all these findings demonstrate that the combination of PARPis with other drugs/inhibitors may expand their application in ovarian cancer with wild-type BRCA status. However, DNMTi (44), cyclin D1 silencing and AKT inhibitors $(14,15)$ have not yet been approved by the FDA, while the use carboplatin is limited due to its side effects and drug resistance (50).

Bazedoxifene has been approved by the FDA as a thirdgeneration selective estrogen receptor modulator for the prevention of postmenopausal osteoporosis, showing minimal side effects (26). Moreover, it acts as an IL-6 inhibitor that downregulates the IL6/STAT3 pathway and downstream target genes, such as p-AKT, c-Myc, p-ERK (4, 18). Bazedoxifene also inhibits the expression of ER $\alpha$, which 


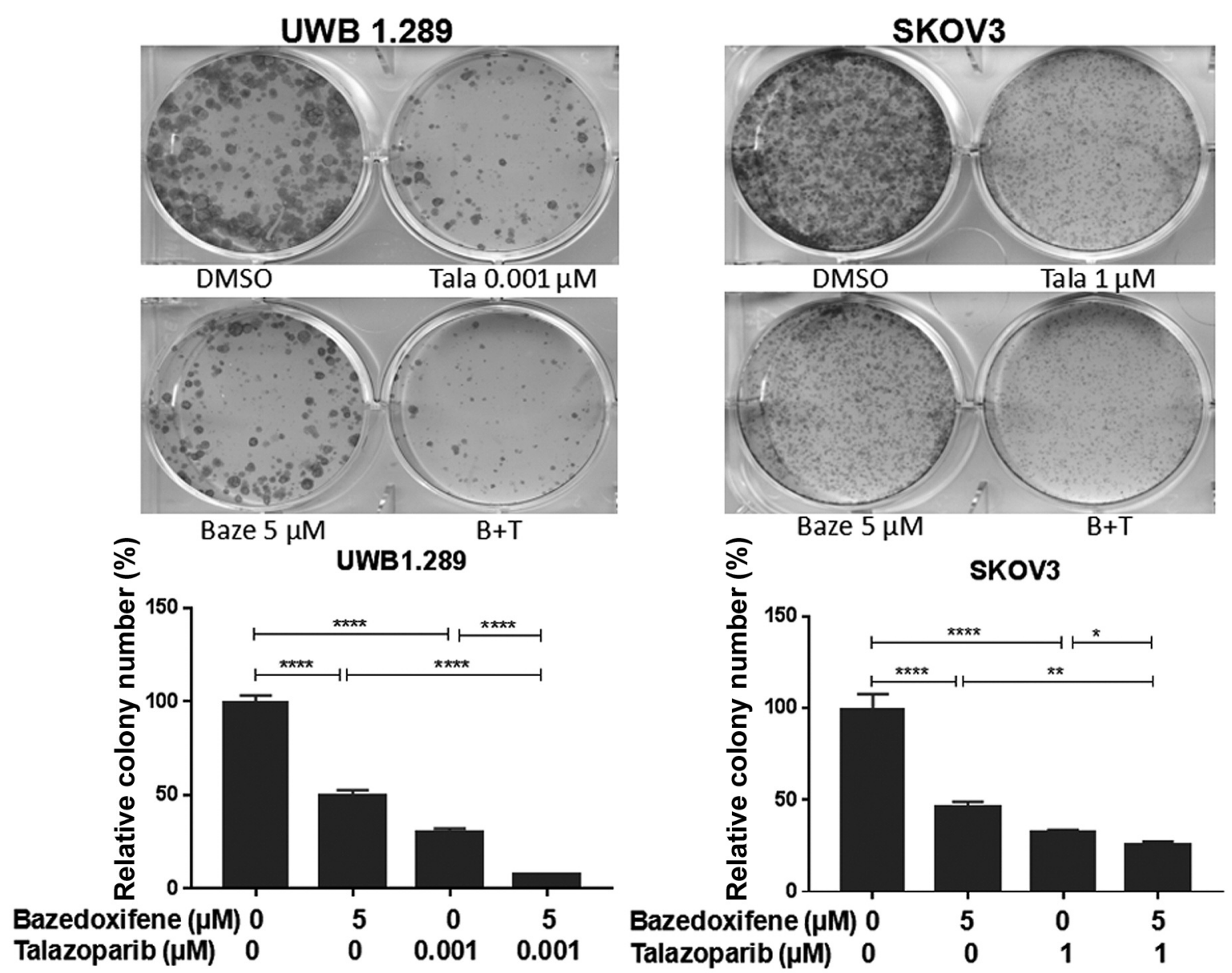

Figure 5. Cell colony formation assay after treatment with varying concentrations of talazoparib and bazedoxifene monotherapy, combination treatment, or DMSO. The combination of bazedoxifene and talazoparib synergistically inhibited colony formation of ovarian cancer cells. ${ }^{*} p<0.05$, $* * p<0.01$ and $* * * * p<0.0001$.

interplays with the IL-6 pathway (29). According to previous studies, bazedoxifene has shown antitumor activity in many cancer types, such as breast cancer (23), colon cancer (30), head and neck cancer (25) and gastrointestinal cancer (51). However, there are few studies on its application in ovarian cancer.

In the present study, the combination of talazoparib and bazedoxifene was studied in BRCA wild-type as well as BRCA-null ovarian cancer cell lines, in vitro. Talazoparib, is a novel FDA-approved PARP inhibitor, which additionally exhibits tumor growth suppression activity through other pathways, such as sex hormone, ERK, and AKT pathways (13). The results showed that cell viability, growth, migration and colony formation were inhibited by talazoparib and bazedoxifene monotherapy, while combination treatment demonstrated a greater inhibitory effect than monotherapy; these findings were also confirmed by western blot. Cell stemness is related to tumor recurrence and metastasis (36,
37) and cell migration is a crucial step in cancer cell metastasis $(52,53)$. Therefore, bazedoxifene and talazoparib combination treatment may have the potential to prevent ovarian cancer cell recurrence and metastasis.

\section{Conclusion}

The combination of IL-6 and PARP inhibitors induced synergistic inhibition of cell viability, cell growth, migration, and colony formation in ovarian cancer cells, regardless of BRCA mutation status. In addition, $\gamma$-H2AX expression was induced by the two drugs, which demonstrates that combination treatment induced double-strand breaks. Thus, this IL-6 inhibitor and PARPi combination has the potential to be a novel combination targeted therapy against ovarian cancer regardless of BRCA mutation status. Further research is warranted to investigate this drug combination as a candidate for targeted anti-cancer therapies. 

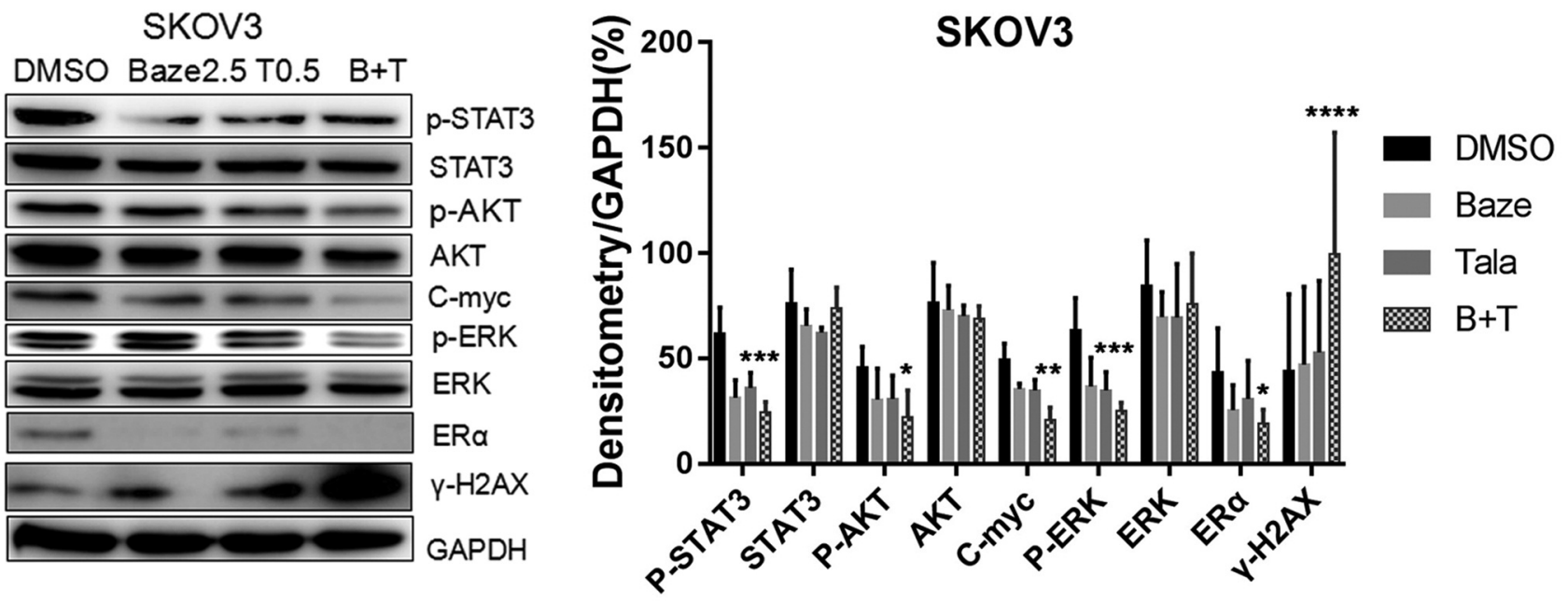

UWB1.289

DMSO Baze2.5 T0.5 B+T

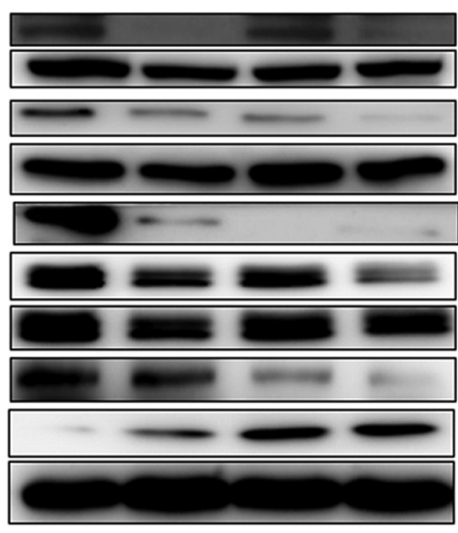

p-STAT3
STAT3
p-AKT
AKT
C-myc
p-ERK
ERK
ERa
Y-H2AX
GAPDH

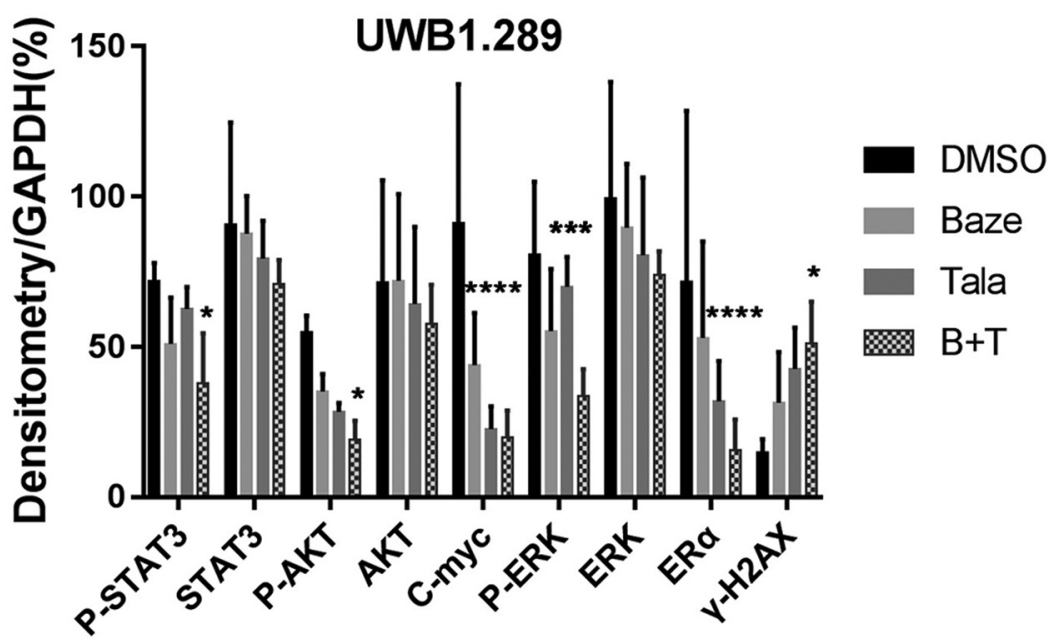

Figure 6. Bazedoxifene combined with talazoparib synergistically inhibited the expression of IL-6 pathway and DNA repair downstream target genes in human ovarian cancer cells. SKOV3 and UWB1.289 cells were treated with talazoparib and bazedoxifene monotherapy, combination treatment, or DMSO. Levels of $p$-STAT3, $p$-AKT, $p$-ERK, $c$-Myc and $\gamma$-H2AX expression were determined by Western blot analysis (B+T group/DMSO control). $* p<0.05, * * p<0.01, * * * p<0.001$ and $* * * p<0.0001$.

\section{Conflicts of Interest}

The Authors declare no conflicts of interest.

\section{Authors' Contributions}

Ruijie Zhang and Jiayuh Lin conceived the original idea. Jiayuh Lin supervised the project. Ruijie Zhang carried out the experiment and wrote the manuscript with support from Jiayuh Lin. Tiffany Wang contributed to data analysis and editing the manuscript.

\section{Acknowledgements}

The Authors thank Dr. Rich Eckert at the Department of Biochemistry and Molecular Biology at the University of Maryland
(Baltimore, MD, USA) for providing microscope access for use for the wound healing assay. This research was supported by the University of Maryland School of Medicine and Comprehensive Cancer Center startup fund.

\section{References}

1 Siegel RL, Miller KD and Jemal A: Cancer statistics, 2019. CA Cancer J Clin 69(1): 7-34, 2019. PMID: 30620402. DOI: 10.3322/caac. 21551

2 Armstrong DK, Alvarez RD, Bakkum-Gamez JN, Barroilhet L, Behbakht K, Berchuck A, Chen LM, Cristea M, DeRosa M, Eisenhauer EL, Gershenson DM, Gray HJ, Grisham R, Hakam A, Jain A, Karam A, Konecny GE, Leath CA, Liu J, Mahdi H, Martin L, Matei D, McHale M, McLean K, Miller DS, O’Malley 
DM, Percac-Lima S, Ratner E, Remmenga SW, Vargas R, Werner TL, Zsiros E, Burns JL and Engh AM: Ovarian cancer, version 2.2020, NCCN clinical practice guidelines in oncology. J Natl Compr Canc Netw 19(2): 191-226, 2021. PMID: 33545690. DOI: $10.6004 /$ jncen.2021.0007

3 Coleman RL, Monk BJ, Sood AK and Herzog TJ: Latest research and treatment of advanced-stage epithelial ovarian cancer. Nat Rev Clin Oncol 10(4): 211-224, 2013. PMID: 23381004. DOI: 10.1038/nrclinonc.2013.5

4 Browning L, Patel MR, Horvath EB, Tawara K and Jorcyk CL: IL-6 and ovarian cancer: inflammatory cytokines in promotion of metastasis. Cancer Manag Res 10: 6685-6693, 2018. PMID: 30584363. DOI: $10.2147 /$ CMAR.S 179189

5 Walsh C: Targeted therapy for ovarian cancer the rapidly evolving landscape of PARP inhibitor use. Minerva Ginecol 70(2): 150-170, 2018. PMID: 28994564. DOI: 10.23736/S00264784.17.04152-1

6 Ledermann J, Harter P, Gourley C, Friedlander M, Vergote I, Rustin G, Scott C, Meier W, Shapira-Frommer R, Safra T, Matei D, Macpherson E, Watkins C, Carmichael $\mathrm{J}$ and Matulonis U: Olaparib maintenance therapy in platinum-sensitive relapsed ovarian cancer. N Engl J Med 366(15): 1382-1392, 2012. PMID: 22452356. DOI: 10.1056/NEJMoa1105535

7 Farmer H, McCabe N, Lord CJ, Tutt AN, Johnson DA, Richardson TB, Santarosa M, Dillon KJ, Hickson I, Knights C, Martin NM, Jackson SP, Smith GC and Ashworth A: Targeting the DNA repair defect in BRCA mutant cells as a therapeutic strategy. Nature 434(7035): 917-921, 2005. PMID: 15829967. DOI: $10.1038 /$ nature03445

8 Bryant HE, Schultz N, Thomas HD, Parker KM, Flower D, Lopez E, Kyle S, Meuth M, Curtin NJ and Helleday T: Specific killing of BRCA2-deficient tumours with inhibitors of poly(ADP-ribose) polymerase. Nature 434(7035): 913-917, 2005. PMID: 15829966. DOI: 10.1038/nature03443

9 Lord CJ and Ashworth A: PARP inhibitors: Synthetic lethality in the clinic. Science 355(6330): 1152-1158, 2017. PMID: 28302823. DOI: $10.1126 /$ science.aam7344

10 Pommier Y, O'Connor MJ and de Bono J: Laying a trap to kill cancer cells: PARP inhibitors and their mechanisms of action. Sci Transl Med 8(362): 362ps17, 2016. PMID: 27797957. DOI: 10.1126/scitranslmed.aaf9246

11 Hoy SM: Talazoparib: First global approval. Drugs 78(18): 1939-1946, 2018. PMID: 30506138. DOI: 10.1007/s40265-0181026-z

12 Hijaz M, Chhina J, Mert I, Taylor M, Dar S, Al-Wahab Z, AliFehmi R, Buekers T, Munkarah AR and Rattan R: Preclinical evaluation of olaparib and metformin combination in BRCA1 wildtype ovarian cancer. Gynecol Oncol 142(2): 323-331, 2016. PMID: 27282964. DOI: 10.1016/j.ygyno.2016.06.005

13 Weaver AN and Yang ES: Beyond DNA repair: Additional functions of PARP-1 in cancer. Front Oncol 3: 290, 2013. PMID: 24350055. DOI: 10.3389/fonc.2013.00290

14 Zhong Q, Hu Z, Li Q, Yi T, Li J and Yang H: Cyclin D1 silencing impairs DNA double strand break repair, sensitizes BRCA1 wildtype ovarian cancer cells to olaparib. Gynecol Oncol 152(1): 157-165, 2019. PMID: 30414739. DOI: 10.1016/ j.ygyno.2018.10.027

15 Wang D, Li C, Zhang Y, Wang M, Jiang N, Xiang L, Li T, Roberts TM, Zhao JJ, Cheng H and Liu P: Combined inhibition of PI3K and PARP is effective in the treatment of ovarian cancer cells with wild-type PIK3CA genes. Gynecol Oncol 142(3): 548556, 2016. PMID: 27426307. DOI: 10.1016/j.ygyno.2016.07.092

16 Feng L, Qi Q, Wang P, Chen H, Chen Z, Meng Z and Liu L: Serum levels of IL-6, IL-8, and IL-10 are indicators of prognosis in pancreatic cancer. J Int Med Res 46(12): 5228-5236, 2018. PMID: 30304975. DOI: 10.1177/0300060518800588

17 Isobe A, Sawada K, Kinose Y, Ohyagi-Hara C, Nakatsuka E, Makino H, Ogura T, Mizuno T, Suzuki N, Morii E, Nakamura K, Sawada I, Toda A, Hashimoto K, Mabuchi S, Ohta T, Morishige K, Kurachi $\mathrm{H}$ and Kimura T: Interleukin 6 receptor is an independent prognostic factor and a potential therapeutic target of ovarian cancer. PLoS One 10(2): e0118080, 2015. PMID: 25658637. DOI: 10.1371/journal.pone.0118080

18 Coward J, Kulbe H, Chakravarty P, Leader D, Vassileva V, Leinster DA, Thompson R, Schioppa T, Nemeth J, Vermeulen J, Singh N, Avril N, Cummings J, Rexhepaj E, Jirström K, Gallagher WM, Brennan DJ, McNeish IA and Balkwill FR: Interleukin-6 as a therapeutic target in human ovarian cancer. Clin Cancer Res 17(18): 6083-6096, 2011. PMID: 21795409. DOI: $10.1158 / 1078-0432 . C C R-11-0945$

19 Kumari N, Dwarakanath BS, Das A and Bhatt AN: Role of interleukin-6 in cancer progression and therapeutic resistance. Tumour Biol 37(9): 11553-11572, 2016. PMID: 27260630. DOI: 10.1007/s 13277-016-5098-7

20 Wang Y, Qu Y, Niu XL, Sun WJ, Zhang XL and Li LZ: Autocrine production of interleukin-8 confers cisplatin and paclitaxel resistance in ovarian cancer cells. Cytokine 56(2): 365-375, 2011. PMID: 21742513. DOI: 10.1016/j.cyto.2011.06.005

21 Zhu X, Shen H, Yin X, Long L, Chen X, Feng F, Liu Y, Zhao P, $\mathrm{Xu}$ Y, Li M, Xu W and Li Y: IL-6R/STAT3/miR-204 feedback loop contributes to cisplatin resistance of epithelial ovarian cancer cells. Oncotarget 8(24): 39154-39166, 2017. PMID: 28388577. DOI: $10.18632 /$ oncotarget.16610

$22 \mathrm{Wu}$ X, Cao Y, Xiao H, Li C and Lin J: Bazedoxifene as a Novel GP130 Inhibitor for Pancreatic Cancer Therapy. Mol Cancer Ther 15(11): 2609-2619, 2016. PMID: 27535971. DOI: 10.1158/1535-7163.MCT-15-0921

23 Tian J, Chen X, Fu S, Zhang R, Pan L, Cao Y, Wu X, Xiao H, Lin HJ, Lo HW, Zhang Y and Lin J: Bazedoxifene is a novel IL6/GP130 inhibitor for treating triple-negative breast cancer. Breast Cancer Res Treat 175(3): 553-566, 2019. PMID: 30852762. DOI: $10.1007 / \mathrm{s} 10549-019-05183-2$

24 Johnson DE, O'Keefe RA and Grandis JR: Targeting the IL6/JAK/STAT3 signalling axis in cancer. Nat Rev Clin Oncol 15(4): 234-248, 2018. PMID: 29405201. DOI: 10.1038/ nrclinonc. 2018.8

25 Yadav A, Kumar B, Teknos TN and Kumar P: Bazedoxifene enhances the anti-tumor effects of cisplatin and radiation treatment by blocking IL- 6 signaling in head and neck cancer. Oncotarget 8(40): 66912-66924, 2016. PMID: 28978005. DOI: 10.18632 /oncotarget.11464

26 Gennari L, Merlotti D, De Paola V, Martini G and Nuti R: Bazedoxifene for the prevention of postmenopausal osteoporosis. Ther Clin Risk Manag 4(6): 1229-1242, 2008. PMID: 19337430. DOI: $10.2147 /$ tcrm.s3476

27 Ma H, Yan D, Wang Y, Shi W, Liu T, Zhao C, Huo S, Duan J, Tao J, Zhai M, Luo P, Guo J, Tian L, Mageta L, Jou D, Zhang C, Li C, Lin J, Lv J, Li S and Lin L: Bazedoxifene exhibits growth suppressive activity by targeting interleukin-6/glycoprotein 130/signal transducer and activator of transcription 3 signaling in 
hepatocellular carcinoma. Cancer Sci 110(3): 950-961, 2019. PMID: 30648776. DOI: 10.1111/cas.13940

28 Komm BS, Kharode YP, Bodine PV, Harris HA, Miller CP and Lyttle CR: Bazedoxifene acetate: a selective estrogen receptor modulator with improved selectivity. Endocrinology 146(9): 3999-4008, 2005. PMID: 15961563. DOI: 10.1210/en.20050030

29 Romero IL, Lee W, Mitra AK, Gordon IO, Zhao Y, Leonhardt P, Penicka CV, Mui KL, Krausz TN, Greene GL and Lengyel E: The effects of $17 \beta$-estradiol and a selective estrogen receptor modulator, bazedoxifene, on ovarian carcinogenesis. Gynecol Oncol 124(1): 134-141, 2012. PMID: 21996264. DOI: 10.1016/j.ygyno.2011.08.026

30 Wei J, Ma L, Lai YH, Zhang R, Li H, Li C and Lin J: Bazedoxifene as a novel GP130 inhibitor for Colon Cancer therapy. J Exp Clin Cancer Res 38(1): 63, 2019. PMID: 30736824. DOI: 10.1186/s13046-019-1072-8

31 Shin HY, Yang W, Lee EJ, Han GH, Cho H, Chay DB and Kim $\mathrm{JH}$ : Establishment of five immortalized human ovarian surface epithelial cell lines via SV40 T antigen or HPV E6/E7 expression. PLoS One 13(10): e0205297, 2018. PMID: 30296284. DOI: 10.1371/journal.pone.0205297

32 DelloRusso C, Welcsh PL, Wang W, Garcia RL, King MC and Swisher EM: Functional characterization of a novel BRCA1-null ovarian cancer cell line in response to ionizing radiation. Mol Cancer Res 5(1): 35-45, 2007. PMID: 17259345. DOI: 10.1158/1541-7786.MCR-06-0234

33 Chou TC: Theoretical basis, experimental design, and computerized simulation of synergism and antagonism in drug combination studies. Pharmacol Rev 58(3): 621-681, 2006. PMID: 16968952 . DOI: $10.1124 /$ pr.58.3.10

$34 \mathrm{Fu} \mathrm{S}$, Chen X, Lin HJ and Lin J: Inhibition of interleukin 8/C-X-C chemokine receptor $1, / 2$ signaling reduces malignant features in human pancreatic cancer cells. Int J Oncol 53(1): 349-357, 2018. PMID: 29749433. DOI: 10.3892/ijo.2018.4389

35 Xiao H, Bid HK, Chen X, Wu X, Wei J, Bian Y, Zhao C, Li H, Li C and Lin J: Repositioning Bazedoxifene as a novel IL6/GP130 signaling antagonist for human rhabdomyosarcoma therapy. PLoS One 12(7): e0180297, 2017. PMID: 28672024. DOI: 10.1371 /journal.pone. 0180297

36 Chang JC: Cancer stem cells: Role in tumor growth, recurrence, metastasis, and treatment resistance. Medicine (Baltimore) 95(1 Suppl 1): S20-S25, 2016. PMID: 27611935. DOI: 10.1097/ MD.0000000000004766

37 Li Y, Rogoff HA, Keates S, Gao Y, Murikipudi S, Mikule K, Leggett D, Li W, Pardee AB and Li CJ: Suppression of cancer relapse and metastasis by inhibiting cancer stemness. Proc Natl Acad Sci USA 112(6): 1839-1844, 2015. PMID: 25605917. DOI: $10.1073 /$ pnas. 1424171112

38 Dickey JS, Redon CE, Nakamura AJ, Baird BJ, Sedelnikova OA and Bonner WM: H2AX: functional roles and potential applications. Chromosoma 118(6): 683-692, 2009. PMID: 19707781. DOI: 10.1007/s00412-009-0234-4

39 Mantia-Smaldone GM, Edwards RP and Vlad AM: Targeted treatment of recurrent platinum-resistant ovarian cancer: current and emerging therapies. Cancer Manag Res 3: 25-38, 2011. PMID: 21734812. DOI: 10.2147/CMR.S8759

$40 \mathrm{Lim}$ HJ and Ledger W: Targeted therapy in ovarian cancer. Womens Health (Lond) 12(3): 363-378, 2016. PMID: 27215391. DOI: $10.2217 /$ whe. 16.4
41 McLachlan J, George A and Banerjee S: The current status of PARP inhibitors in ovarian cancer. Tumori 102(5): 433-440, 2016. PMID: 27716873. DOI: 10.5301/tj.5000558

42 Zheng F, Zhang Y, Chen S, Weng X, Rao Y and Fang H: Mechanism and current progress of Poly ADP-ribose polymerase (PARP) inhibitors in the treatment of ovarian cancer. Biomed Pharmacother 123: 109661, 2020. PMID: 31931287. DOI: 10.1016/j.biopha.2019.109661

43 Walsh CS: Latest clinical evidence of maintenance therapy in ovarian cancer. Curr Opin Obstet Gynecol 32(1): 15-21, 2020. PMID: 31833941. DOI: 10.1097/GCO.0000000000000592

44 Pulliam N, Fang F, Ozes AR, Tang J, Adewuyi A, Keer H, Lyons J, Baylin SB, Matei D, Nakshatri H, Rassool FV, Miller KD and Nephew KP: An effective epigenetic-PARP inhibitor combination therapy for breast and ovarian cancers independent of BRCA mutations. Clin Cancer Res 24(13): 3163-3175, 2018. PMID: 29615458. DOI: 10.1158/1078-0432.CCR-18-0204

45 Ledermann JA and Pujade-Lauraine E: Olaparib as maintenance treatment for patients with platinum-sensitive relapsed ovarian cancer. Ther Adv Med Oncol 11: 1758835919849753, 2019. PMID: 31205507. DOI: 10.1177/1758835919849753

46 Scott LJ: Niraparib: First global approval. Drugs 77(9): 1029-1034, 2017. PMID: 28474297. DOI: 10.1007/s40265-017-0752-y

47 O'Connor MJ: Targeting the DNA damage response in cancer. Mol Cell 60(4): 547-560, 2015. PMID: 26590714. DOI: 10.1016/j.molcel.2015.10.040

48 Syed YY: Rucaparib: First global approval. Drugs 77(5): 585592, 2017. PMID: 28247266. DOI: 10.1007/s40265-017-0716-2

49 Shen YT, Evans JC, Zafarana G, Allen C and Piquette-Miller M: BRCA status does not predict synergism of a carboplatin and olaparib combination in high-grade serous ovarian cancer cell lines. Mol Pharm 15(7): 2742-2753, 2018. PMID: 29750868. DOI: 10.1021/acs.molpharmaceut.8b00246

50 McGuire WP 3rd and Markman M: Primary ovarian cancer chemotherapy: current standards of care. Br J Cancer 89 Suppl 3: S3-S8, 2003. PMID: 14661040. DOI: 10.1038/sj.bjc.6601494

51 Thilakasiri P, Huynh J, Poh AR, Tan CW, Nero TL, Tran K, Parslow AC, Afshar-Sterle S, Baloyan D, Hannan NJ, Buchert M, Scott AM, Griffin MD, Hollande F, Parker MW, Putoczki TL, Ernst $M$ and Chand AL: Repurposing the selective estrogen receptor modulator bazedoxifene to suppress gastrointestinal cancer growth. EMBO Mol Med 11(4): e9539, 2019. PMID: 30885958. DOI: $10.15252 / \mathrm{emmm} .201809539$

53 Hall DM and Brooks SA: In vitro invasion assay using matrige $^{\mathrm{TM}}$ : a reconstituted basement membrane preparation. Methods Mol Biol 1070: 1-11, 2014. PMID: 24092428. DOI: 10.1007/978-1-4614-8244-4_1

53 Bravo-Cordero JJ, Hodgson L and Condeelis J: Directed cell invasion and migration during metastasis. Curr Opin Cell Biol 24(2): 277-283, 2012. PMID: 22209238. DOI: 10.1016/ j.ceb.2011.12.004 